\title{
Experimental validation of a novel angular estimator for synthetic inertia support under disturbed network conditions
}

\author{
Alberto Bolzoni, Roberto Perini \\ POLITECNICO DI MILANO \\ via La Masa 34, \\ 20156 Milano, Italy \\ alberto.bolzoni@polimi.it, roberto.perini@polimi.it
}

\section{Keywords}

«Control methods for electrical systems», «Estimation technique», «Converter control», «Microgrids»

\begin{abstract}
This paper proposes a novel estimation scheme for angular quantities extraction under disturbed network conditions; a robust identification of the network angular frequency and of its derivative are crucial for the synthetic inertia provision from grid-connected converters. The newly proposed architecture is derived from the Second Order / Second Order Generalized Integrator scheme (SOSOGI), an algorithm exploited to extract the fundamental harmonic component from phase voltages measurements, with the introduction of an additional decoupling feedback for negative sequence compensation. The effectiveness and robustness of the proposed technique are compared, both analytically and experimentally, with other schemes already available in literature.
\end{abstract}

\section{Introduction}

In recent years, an increasing interest in frequency support from grid-connected converters has raised. Beside primary frequency regulation from electrochemical storage units equipped with droop control [1] - [2], a new up-to-date paradigm is represented by the provision of transitory inertia support from non-dispatchable power sources to reduce the overshoot during the first instants after a severe frequency transient (synthetic inertia).

Even though several synthetic inertia schemes have been proposed in literature ([3] - [5]), all the architectures imply a fast and clean estimation of the grid angular quantities (angular frequency and its derivative) from the available voltage measurements, even under disturbed network conditions. A typical solution adopted to reduce the estimation sensitivity consists in the application of a strong lowpass filter on the reconstructed states to improve the signal-to-noise ratio, even though this may jeopardize the speed of the reconstruction itself due to the slow filter's dynamics.

The development of advanced measurement apparatus (like Phasor Measurement Units - PMUs) [6] represents only a partial solution to the problem due to the significant cost of these devices, which prevents a widespread application to small-size distributed generators. Thus, in this paper, a novel control scheme is proposed for local angular quantities reconstruction in grid-connected converters: the scheme is obtained from a Second-Order / Second-Order Generalized Integrator (SO-SOGI) [7] equipped with Frequency Locked Loop (FLL) and extended with an additional feedback for negative sequence disturbance reduction (SO-SOGI-N). The design of the algorithm internal dynamics is developed referring to resonant controllers rather than typical DSOGI coupling feedbacks [8]: this significantly simplifies the parameters definition and guarantees coherent dynamical interaction between the algorithm sub-functions. The performances of the proposed architecture will be assessed against the well-known DSOGI scheme [8], briefly recalled in the next section for clarity, and against the typical SO-SOGI algorithm [7].

\section{DSOGI architecture: a review}

\section{DSOGI system}

In this first section, the three-phase DSOGI control is recalled. This serves as a background for the 
development of the proposed SO-SOGI-N scheme, illustrated in the next sections. Instead of referring to the typical nested-feedbacks configuration [8], a simpler implementation based on equivalent realcoefficients resonant controllers $D(s)$ and $Q(s)$ can be introduced, according to the scheme reported in Figure 1.a: this simplifies the physical interpretation of the algorithm and allows to quantify its interactions with the external slower loops. The following notation is adopted in this paper:

- $\omega_{n}$ : resonance angular frequency of the controllers $D(s)$ and $Q(s)$;

- $\quad \xi$ : damping factor of the controllers $D(s)$ and $Q(s)$;

- $\omega_{1}$ : network fundamental angular frequency $(2 \pi \cdot 50 \mathrm{rad} / \mathrm{s}$ in Europe);

- $s=j \omega_{s}$ : Laplace operator and symbolic frequency response variable.
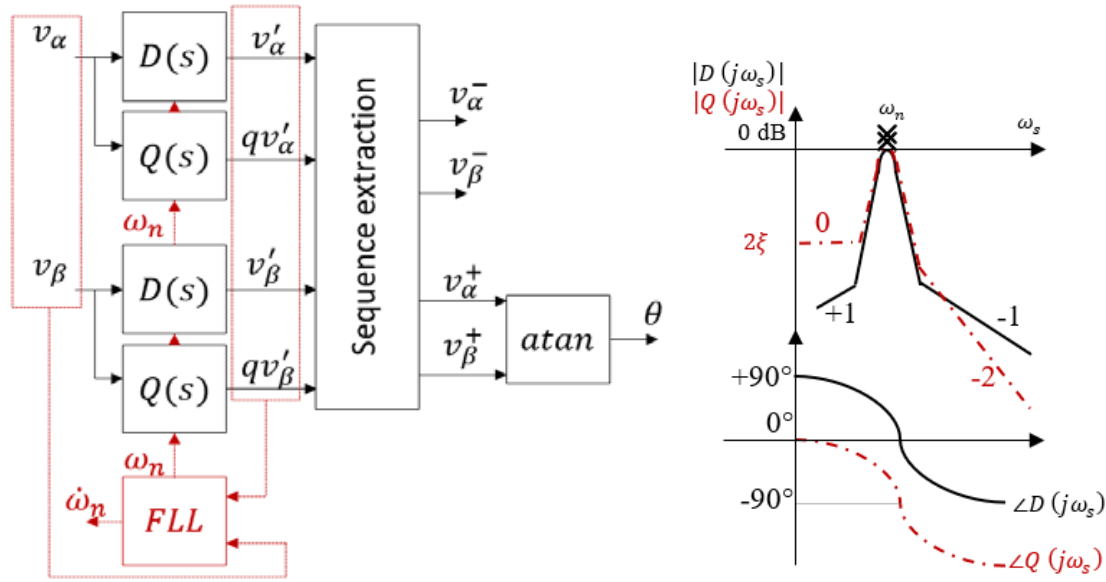

Figure 1: (a) DSOGI scheme and (b) resonant transfer function $D(s)$ and $Q(s)$.

Consider the expressions of the $\alpha \beta$ components of a three-phase voltage set in the Park domain, under disturbed grid conditions (1). The signals $\left(v_{\alpha}^{\prime}, v_{\beta}^{\prime}\right)$ and $\left(q v_{\alpha}^{\prime}, q v_{\beta}^{\prime}\right)$ are respectively obtained filtering the inputs by $D(s)$ and $Q(s)$ :

$$
\begin{aligned}
& v_{\alpha}+j v_{\beta}=\sum_{h=1}^{N+} \widehat{V}_{h}^{+} \cdot e^{j h \omega_{1} t}+\sum_{h=1}^{N-} \widehat{V}_{h}^{-} \cdot e^{-j h \omega_{1} t} \\
& D(s)=2 \xi \cdot \frac{\frac{s}{\omega_{n}}}{\left(\frac{s}{\omega_{n}}\right)^{2}+2 \xi \frac{s}{\omega_{n}}+1} \quad Q(s)=2 \xi \cdot \frac{1}{\left(\frac{s}{\omega_{n}}\right)^{2}+2 \xi \frac{s}{\omega_{n}}+1}
\end{aligned}
$$

$v_{\alpha}^{\prime}+j v_{\beta}^{\prime}=\sum_{h=1}^{N+} \widehat{V}_{h}^{+} \cdot\left|D\left(j h \omega_{1}\right)\right| e^{j\left[h \omega_{1} t+\measuredangle D\left(j h \omega_{1}\right)\right]}+\sum_{h=1}^{N-} \widehat{V}_{h}^{-} \cdot\left|D\left(j h \omega_{1}\right)\right| e^{-j\left[h \omega_{1} t+\measuredangle D\left(j h \omega_{1}\right)\right]}$

$q v_{\alpha}^{\prime}+j q v_{\beta}^{\prime}=\sum_{h=1}^{N+} \widehat{V}_{h}^{+}\left|Q\left(j h \omega_{1}\right)\right| e^{j\left[h \omega_{1} t+\Varangle Q\left(j h \omega_{1}\right)\right]}+\sum_{h=1}^{N-} \widehat{V}_{h}^{-}\left|Q\left(j h \omega_{1}\right)\right| e^{-j\left[h \omega_{1} t+\Varangle Q\left(j h \omega_{1}\right)\right]}$

where the frequency response magnitudes and phase displacements (Fig. 1-(b)) at the generalized angular frequency $\omega_{s}=h \omega_{1}$ are given by:

$$
\begin{array}{ll}
\left|D\left(j \omega_{s}\right)\right|=\left|\frac{j 2 \xi \omega_{s} \omega_{n}}{\sqrt{\left(\omega_{n}^{2}-\omega_{s}^{2}\right)^{2}+\left(2 \xi \omega_{s} \omega_{n}\right)^{2}}}\right| & \measuredangle D\left(j \omega_{s}\right)=\operatorname{atan}\left(\frac{\omega_{n}^{2}-\omega_{s}^{2}}{2 \xi \omega_{n} \omega_{s}}\right) \\
\left|Q\left(j \omega_{s}\right)\right|=\left|D\left(j \omega_{s}\right)\right| \cdot \frac{\omega_{s}}{\omega_{n}} & \Varangle Q\left(j \omega_{s}\right)=\Varangle D\left(j \omega_{s}\right)-\frac{\pi}{2}
\end{array}
$$

As the relative displacement between the frequency responses of $D(s)$ and $Q(s)$ is constantly equal to $90^{\circ}$ in the whole frequency range (Fig. 1.b), the obtained signals $\left(v_{\alpha}^{\prime}, v_{\beta}^{\prime}, q v_{\alpha}^{\prime}, q v_{\beta}^{\prime}\right)$ allows to calculate the positive $\left(v_{\alpha}^{+}, v_{\beta}^{+}\right)$and negative $\left(v_{\alpha}^{-}, v_{\beta}^{-}\right)$sequence components of the input voltages 
$\left(v_{\alpha}, v_{\beta}\right)$ according to the equalities reported in (7); this operation is represented by the "Sequence extraction" block in Fig. 1.a. Even though these equations have already been proposed in literature [8], it is often difficult to provide an intuitive understanding of their applicability; the Appendix at the end of the paper provides a rigorous proof of their derivation process.

$$
v_{\alpha}^{+}=\frac{v_{\alpha}^{\prime}-q v_{\beta}^{\prime}}{2} \quad v_{\beta}^{+}=\frac{q v_{\alpha}^{\prime}+v_{\beta}^{\prime}}{2} \quad v_{\alpha}^{-}=\frac{v_{\alpha}^{\prime}+q v_{\beta}^{\prime}}{2} \quad v_{\beta}^{-}=\frac{-q v_{\alpha}^{\prime}+v_{\beta}^{\prime}}{2}
$$

\section{DSOGI coordination with the FLL algorithm}

To guarantee that the resonance frequency $\omega_{n}$ converges to the fundamental $\omega_{1}$, an external adaptive feedback referred as Frequency Locked Loop (FLL) is introduced. Its inputs and outputs are represented in Fig. 1.a, while the internal control scheme is reported in Fig. 2. According to [8], the FLL behaves as a first order unitary-gain transfer function, whose passband is determined by the parameter $k_{F L L}$ :

$$
\omega_{n}=\frac{k_{F L L}}{s+k_{F L L}} \omega_{1} \quad \dot{\omega}_{n}=\frac{s \cdot k_{F L L}}{s+k_{F L L}} \omega_{1}
$$

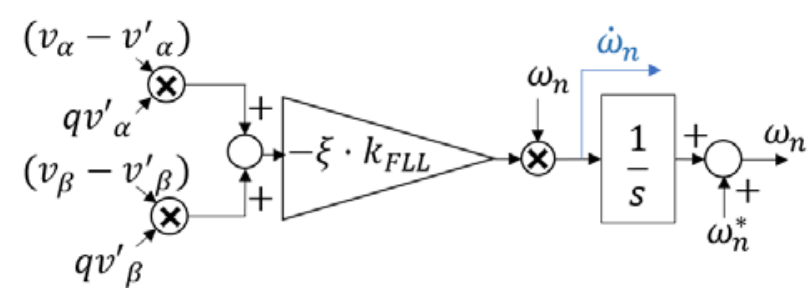

Figure 2: Frequency Locked Loop for the DSOGI scheme.

\section{Weaknesses of the DSOGI architecture}

The main criticalities associated to the DSOGI scheme are the following:

- poor disturbance rejection of the function $Q(s)$ in the frequency range before the resonance $\omega_{n}$;

- limited rejection of the function $D(s)$ for disturbance components above $\omega_{n}$;

- high sensitivity of the estimation with respect to the first-order negative sequence component, which is not attenuated by either $D(s)$ or $Q(s)$;

In order to solve the first two issues, a modified architecture is proposed in [7]: this goes under the name of Second Order / Second Order Generalized Integrator (SO-SOGI) and combines the good rejection properties of $D(s)$ at low frequency with the ones of $Q(s)$ in the higher part pf the spectrum to improve the signal-to-noise ratio of the estimation. Nevertheless, as for the Authors' knowledge, a detailed analysis of the SO-SOGI coordination with the FLL loop is not available in literature and no solution is proposed to heal its sensitivity with respect to the first-order negative sequence: these are the key aspects introduced by the SO-SOGI-N scheme proposed in this paper.

\section{Proposed SO-SOGI-N architecture}

\section{SO-SOGI-N scheme}

Consider the scheme reported in Figure 3.a, which represents the structure of the proposed threephase SO-SOGI-N system; similarly to the DSOGI architecture, the input is constituted by the $\alpha \beta$ components of the grid voltage space vector $\bar{v}=v_{\alpha}+j v_{\beta}$. Nevertheless, the quadrature components $\left(q v_{\alpha}^{\prime}+j q v_{\beta}^{\prime}\right)$ are obtained filtering the input signals $\left(v_{\alpha}+j v_{\beta}\right)$ by both $D(s)$ and $Q(s)$. Given the different dynamical interaction with respect to the DSOGI scheme, the structure of the FLL (Fig. 3.b) should be modified with respect to the one introduced in Fig. 2; furthermore, an additional decoupling feedback is introduced to heal the algorithm sensitivity with respect to the negative sequence firstorder component. These two aspects will be analysed separately in the next sub-sections. 


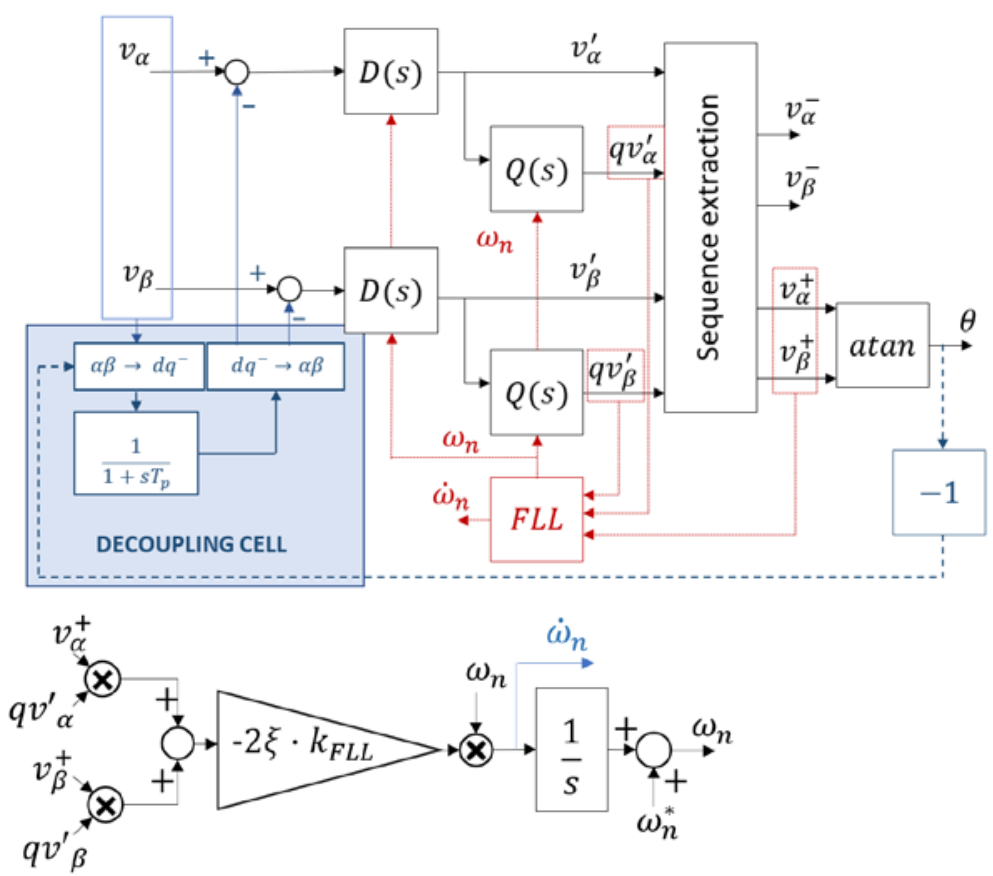

Figure 3: (a) SO-SOGI-N scheme for angular quantities extraction and (b) its FLL algorithm.

\section{Coordination of the SO-SOGI-N with the FLL algorithm}

If we refer to the SO-SOGI topology in Fig. 3.a, the expression associated to the filtered quadrature space vector $\left(q v_{\alpha}^{\prime}+j q v_{\beta}^{\prime}\right)$ can be derived (9) combining the frequency responses of the functions $D(s)$ and $Q(s)$, under the typical input expressed by (1). As regards the in-phase space vector $\left(v_{\alpha}^{\prime}+j v_{\beta}^{\prime}\right)$, its expression is the same already reported in (3).

$$
\begin{aligned}
q v_{\alpha}^{\prime}+j q v_{\beta}^{\prime} & =\sum_{h=1}^{N+} \widehat{V}_{h}^{+} \cdot\left|D\left(j h \omega_{1}\right)\right|\left|Q\left(j h \omega_{1}\right)\right| e^{j\left[h \omega_{1} t+\Varangle D\left(j h \omega_{1}\right)+\Varangle Q\left(j h \omega_{1}\right)\right]}+ \\
& +\sum_{h=1}^{N-} \hat{V}_{h}^{-} \cdot\left|D\left(j h \omega_{1}\right)\right|\left|Q\left(j h \omega_{1}\right)\right| e^{-j\left[h \omega_{1} t+\Varangle D\left(j h \omega_{1}\right)+\Varangle Q\left(j h \omega_{1}\right)\right]}
\end{aligned}
$$

As (9) combines both the rejection properties of $D(s)$ and $Q(s)$ in the low and high frequency range, the amplitudes of the components with $h \neq 1$ are minimised; thus (2) and (9) can be respectively simplified as:

$$
\begin{gathered}
v_{\alpha}^{\prime}+j v_{\beta}^{\prime} \cong \widehat{V}_{1}^{+} \cdot\left|D\left(j \omega_{1}\right)\right| e^{j\left[\omega_{1} t+\measuredangle D\left(j \omega_{1}\right)\right]}+\widehat{V}_{1}^{-} \cdot\left|D\left(j \omega_{1}\right)\right| e^{-j\left[\omega_{1} t+\measuredangle D\left(j \omega_{1}\right)\right]} \\
q v_{\alpha}^{\prime}+j q v_{\beta}^{\prime} \cong\left|D\left(j \omega_{1}\right)\right|\left|Q\left(j \omega_{1}\right)\right|\left(\widehat{V}_{1}^{+} e^{j\left[\omega_{1} t+\measuredangle D\left(j \omega_{1}\right)+\measuredangle Q\left(j \omega_{1}\right)\right]}+\widehat{V}_{1}^{-} e^{-j\left[\omega_{1} t+\measuredangle D\left(j \omega_{1}\right)+\measuredangle Q\left(j \omega_{1}\right)\right]}\right)
\end{gathered}
$$

Substituting (10)-(11) in the sequences extraction formulas (7) leads, after simple trigonometric manipulations, to the expressions of $v_{\alpha}^{+}, v_{\beta}^{+}, v_{\alpha}^{-}, v_{\beta}^{-}$reported in (12)-(13); considering the transfer functions shapes (Fig. 1.b) and the fact that the FLL adapts the resonance frequency $\omega_{n}$ to be close to the fundamental $\omega_{1},\left|D\left(j \omega_{1}\right)\right|=\left|Q\left(j \omega_{1}\right)\right|=1$ is assumed in the calculation. Nevertheless, the information related to the phase displacements $\measuredangle D\left(j \omega_{1}\right)$ and $\measuredangle Q\left(j \omega_{1}\right)$ is retained in (12)-(13) as it represents the input of the FLL adaptation process and it allows to determine its dynamical properties. Coherently with the introduced notation, $V_{1}^{+}$and $V_{1}^{-}$stand for the positive / negative sequence space vectors magnitudes while $\varphi_{1}^{+} / \varphi_{1}^{-}$are the corresponding phases. 


$$
\begin{aligned}
v_{\alpha}^{+} \cong+V_{1}^{+} \cos \left(\omega_{1} t\right. & \left.+\frac{3}{2} \measuredangle D\left(j \omega_{1}\right)+\varphi_{1}^{+}\right) \cos \left(\frac{\measuredangle D\left(j \omega_{1}\right)}{2}\right)+ \\
- & V_{1}^{-} \sin \left(\omega_{1} t+\frac{3}{2} \measuredangle D\left(j \omega_{1}\right)+\varphi_{1}^{-}\right) \sin \left(\frac{\not D\left(j \omega_{1}\right)}{2}\right) \\
v_{\beta}^{+} \cong+V_{1}^{+} \sin \left(\omega_{1} t+\frac{3}{2} \measuredangle\right. & \left.D\left(j \omega_{1}\right)+\varphi_{1}^{+}\right) \cos \left(\frac{\measuredangle D\left(j \omega_{1}\right)}{2}\right)+ \\
& +V_{1}^{-} \cos \left(\omega_{1} t+\frac{3}{2} \measuredangle D\left(j \omega_{1}\right)+\varphi_{1}^{-}\right) \sin \left(\frac{\Varangle D\left(j \omega_{1}\right)}{2}\right)
\end{aligned}
$$

The input of the FLL $\left(v_{\alpha}^{+} \cdot q v_{\alpha}^{\prime}+v_{\beta}^{+} \cdot q v_{\beta}^{\prime}\right)$ is modified with respect to the one adopted for the DSOGI scheme, as shown in Fig. 3.b. Under the typical case $V_{1}^{+} \gg V_{1}^{-}$, equations (14)-(15) hold and thus the FLL input is given by (16).

$$
\begin{gathered}
v_{\alpha}^{+} \cdot q v_{\alpha}^{\prime} \cong \frac{V_{1}^{+^{2}}}{2} \cdot \cos \left(\frac{\measuredangle D\left(j \omega_{1}\right)}{2}\right) \cdot\left[\sin \left(\frac{\measuredangle D\left(j \omega_{1}\right)}{2}\right)+\sin \left(2 \omega_{1} t+\frac{7}{2} \measuredangle D\left(j \omega_{1}\right)+2 \varphi_{1}^{+}\right)\right] \\
v_{\beta}^{+} \cdot q v_{\beta}^{\prime} \cong \frac{V_{1}^{+2}}{2} \cdot \cos \left(\frac{\not D\left(j \omega_{1}\right)}{2}\right) \cdot\left[\sin \left(\frac{\measuredangle D\left(j \omega_{1}\right)}{2}\right)-\sin \left(2 \omega_{1} t+\frac{7}{2} \measuredangle D\left(j \omega_{1}\right)+2 \varphi_{1}^{+}\right)\right] \\
\left(v_{\alpha}^{+} \cdot q v_{\alpha}^{\prime}+v_{\beta}^{+} \cdot q v_{\beta}^{\prime}\right)=V_{1}^{+^{2}} \cdot \cos \left(\frac{\not D\left(j \omega_{1}\right)}{2}\right) \cdot \sin \left(\frac{\measuredangle D\left(j \omega_{1}\right)}{2}\right)
\end{gathered}
$$

The linearization of expressed (16) can be obtained recognizing that $\measuredangle D\left(j \omega_{1}\right)$ is small, thus the corresponding asymptotic expressions can be invoked for the trigonometric functions.

$$
\left(v_{\alpha}^{+} \cdot q v_{\alpha}^{\prime}+v_{\beta}^{+} \cdot q v_{\beta}^{\prime}\right) \simeq V_{1}^{+^{2}} \cdot 1 \cdot \frac{\measuredangle D\left(j \omega_{1}\right)}{2} \simeq \frac{V_{1}^{+^{2}}}{2} \cdot \operatorname{atan}\left(\frac{\omega_{n}^{2}-\omega_{1}^{2}}{2 \xi \omega_{n} \omega_{1}}\right)
$$

As the FLL itself imposes $\omega_{n} \cong \omega_{1}$, the asymptotic behaviour for the inverse tangent function leads to (18), where almost unitary amplitude of the fundamental positive-sequence Park voltage is assumed (in per-unit). Note that the normalization coefficients in the FLL schemes for the SO-SOGI-N architecture (Fig. 3.b) modifies with respect to the DSOGI ones (Fig. 2) as a consequence of the change in the $D(s)-Q(s)$ combination, as well as of the different inputs' definition.

$$
\begin{aligned}
v_{\alpha}^{+} \cdot q v_{\alpha}^{\prime}+v_{\beta}^{+} \cdot q v_{\beta}^{\prime} \simeq \frac{\operatorname{atan}\left(\frac{\omega_{n}^{2}-\omega_{1}^{2}}{2 \xi \omega_{n} \omega_{1}}\right)}{2} \simeq \frac{\omega_{n}^{2}-\omega_{1}^{2}}{4 \xi \omega_{n} \omega_{1}} \simeq \\
\simeq \frac{\left(\omega_{n}+\omega_{1}\right)\left(\omega_{n}-\omega_{1}\right)}{4 \xi \omega_{n} \omega_{1}} \simeq \frac{2 \omega_{1}\left(\omega_{n}-\omega_{1}\right)}{4 \xi \omega_{n} \omega_{1}} \simeq \frac{\omega_{n}-\omega_{1}}{2 \xi \omega_{n}}
\end{aligned}
$$

The linearized expression (18) is exploited to tune the normalization coefficients in the loop reported in Fig. 4; by solving the feedback, it is easy to show that the FLL behaves as a first order low-pass filter for the network angular frequency and its derivative (19), where the control parameters $k_{F L L}$ determines the estimation pass-band. A feedforward compensation term $\omega_{n}^{*}=1 p . u$. is introduced to speed up the algorithm convergence at the first computation steps after the initialization, even though this does not affect the dynamical stability properties.

$$
\omega_{n}=\frac{k_{F L L}}{s+k_{F L L}} \omega_{1} \quad \omega_{n}=\frac{s \cdot k_{F L L}}{s+k_{F L L}} \omega_{1}
$$




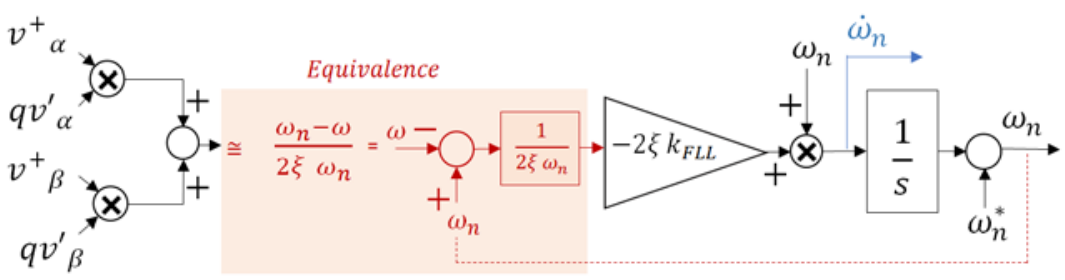

Figure 4: Linearized equivalence of the FLL algorithm for SO-SOGI-N scheme.

\section{Negative sequence decoupling cell}

A further estimation improvement can be obtained introducing a decoupling feedback to reduce the impact of the first-order negative term. Consider the scheme in Fig. 3.a: from the positive sequence $\alpha \beta$ components as extracted by the "Sequence extraction" block, it is possible to calculate the space vector phase angle $\theta$ by means of the inverse tangent function and, subsequently, define an inverse rotating (clockwise) frame by means of the rotation blocks reported in Fig. 3.a $\left(\alpha \beta \rightarrow d q^{-}\right.$and $d q^{-} \rightarrow \alpha \beta$ ). This frame results synchronous with the first-order negative component even under nonnominal network conditions. The interposition of a low-pass filter with high time constant $\left(T_{p}=0.1 \mathrm{~s}\right)$ allows to extract the negative sequence component and enables its deletion from the input of the SOSOGI architecture: this significantly reduces the estimator disturbance sensitivity without affecting its convergence speed, as the quantities of interest (related to the positive sequence) result practically unaffected by the compensation.

\section{Experimental validation}

In order to test the proposed algorithm under controlled and repeatable conditions, the experimental set-up reported in Fig. 5.a has been developed. Two converters are connected in parallel to a highimpedance resistive load:

- the first unit is operated in grid-forming mode and imposes a pre-determined frequency transient on the small-scale microgrid;

- the second converter is operated in PQ mode (grid-following) and acquires a set of three-phase voltages from the grid: the analysed estimation algorithms are coded in the control of this converter and can thus be compared.

As the network frequency is imposed by the grid-forming unit, its time profile is known and replicable (labelled as "real" in the reported results, compared to the "measured" ones obtained by the considered reconstruction algorithms). The assessment refers to two different conditions:

- in steady-state operations, the system angular frequency imposed by the grid-forming converter remains stable. This allows to estimate the Signal-to-Noise ratio for the reconstructed quantities.

- in transitory conditions, the grid-forming converter imposes a known frequency transient to the network, thus it is easy to verify the convergence time of the estimation to the real angular quantity.
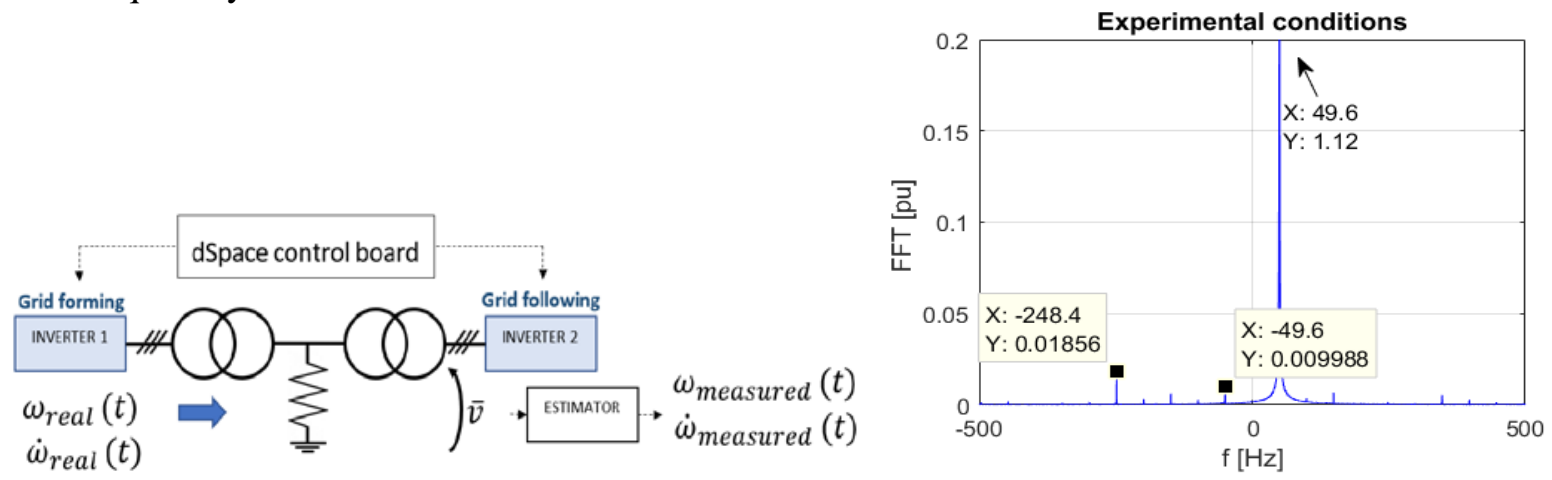

Figure5: (a) Experimental setup and (b) frequency spectrum of the experimental voltages. 
Table I: Design parameters for the experimental test

\begin{tabular}{lll}
\hline Parameter & Symbol & Value \\
\hline Damping factor & $\xi$ & 0.2 \\
Slow FLL & $k_{F L L}$ (slow) & $8 \mathrm{rad} / \mathrm{s}$ \\
Fast FLL & $k_{F L L}$ (fast) & $80 \mathrm{rad} / \mathrm{s}$ \\
Decoupling filter time constant & $T_{p}$ & $0.1 \mathrm{~s}$ \\
\hline
\end{tabular}

Figure 5.b reports the spectral profiles of the steady-state voltages; the steady-state actual frequency $(49.6 \mathrm{~Hz})$ has been taken different from the nominal one $\left(f_{n}^{*}=50 \mathrm{~Hz}\right)$ in order to test the robustness of the algorithms. The spectral diagram shows the presence of an inverse sequence $(1 \%$ of the fundamental) and a $5^{\text {th }}$ order component produced by the physical non-linearities of the experimental setup ( $2 \%$ of the fundamental). Even though these disturbances are limited, they considerably affect the estimation of the angular frequency derivative.

Consider the time profiles associated to a traditional DSOGI algorithm with a fast FLL loop, as reported in Fig, 6: the algorithm correctly estimates the angular frequency, even though the reconstructed derivative profile is unacceptably affected by the disturbance components associated to the negative sequence and $5^{\text {th }}$ order harmonic. A possible solution consists in the reduction of the equivalent FLL bandpass (Fig. 7): the improvements in terms of harmonic content are nullified by the increase of the convergence time, which may be detrimental for the converter response during the provision of regulation services [3]. The SO-SOGI scheme (Fig.8) guarantees acceptable rejection of the disturbance associated to the $5^{\text {th }}$ harmonic even under a fast estimation passband, but still retains a significant sensitivity with respect to the first-order negative sequence term. On the other hand, the proposed SO-SOGI-N architecture (Fig. 9) combines a sufficient estimation fastness and good disturbance rejection, both for the angular frequency and its derivative.
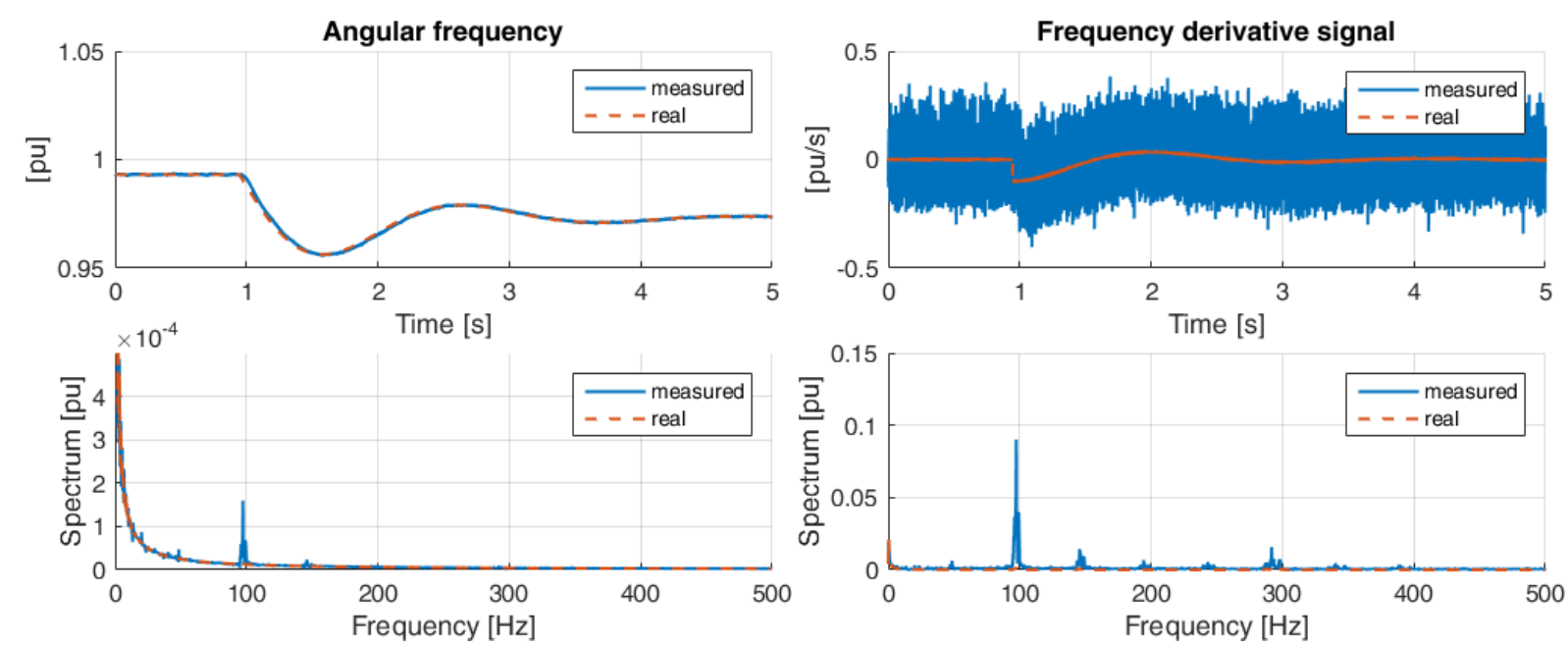

Figure 6: Angular frequency (a) and its derivative (b) obtained by a DSOGI scheme with fast FLL loop $\left(K_{F L L}=80 \mathrm{rad} / \mathrm{s}\right)$. Even though the transitory algorithm convergence is fast, the reconstructed signals are strongly affected by disturbances. 

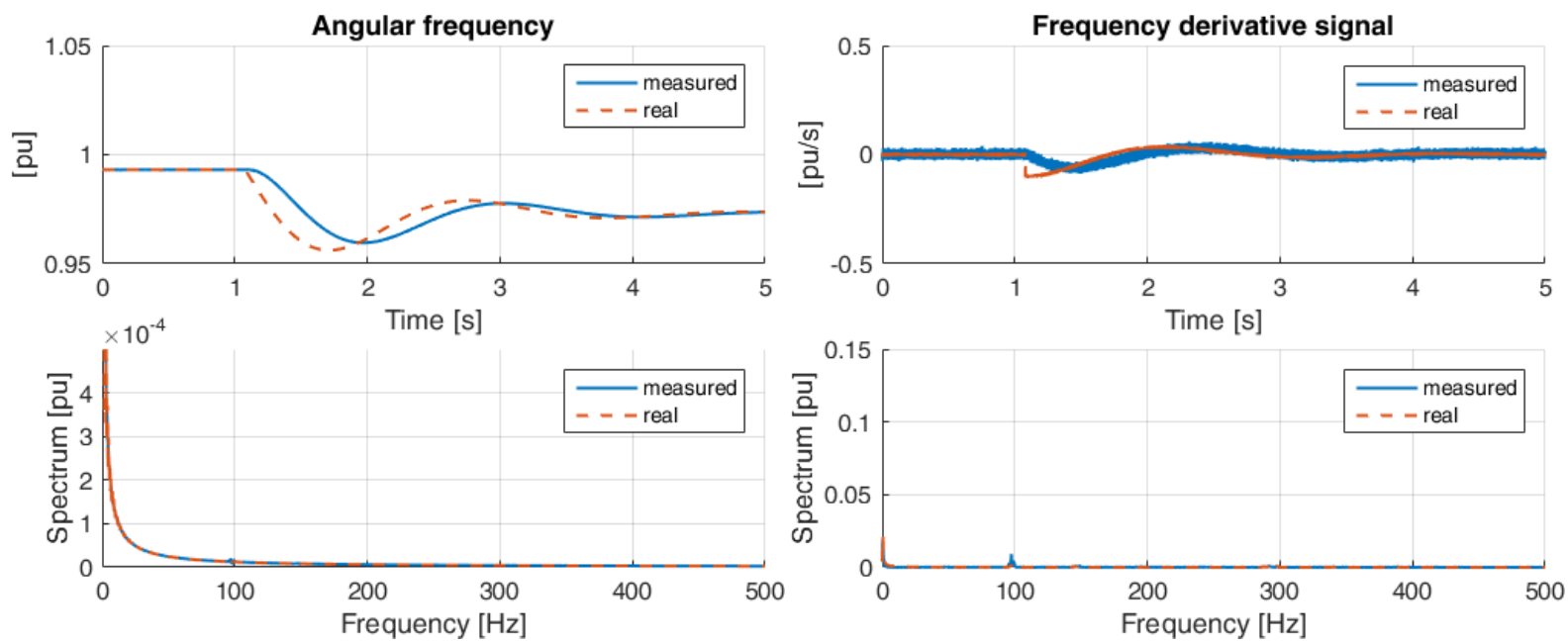

Figure 7: Angular frequency (a) and its derivative (b) obtained by a DSOGI scheme with slow FLL loop $\left(K_{F L L}=8 \mathrm{rad} / \mathrm{s}\right)$. The signal-to-noise ratio is acceptable, but the effect of a slow FLL dynamic produces a significant delay in the reconstruction.
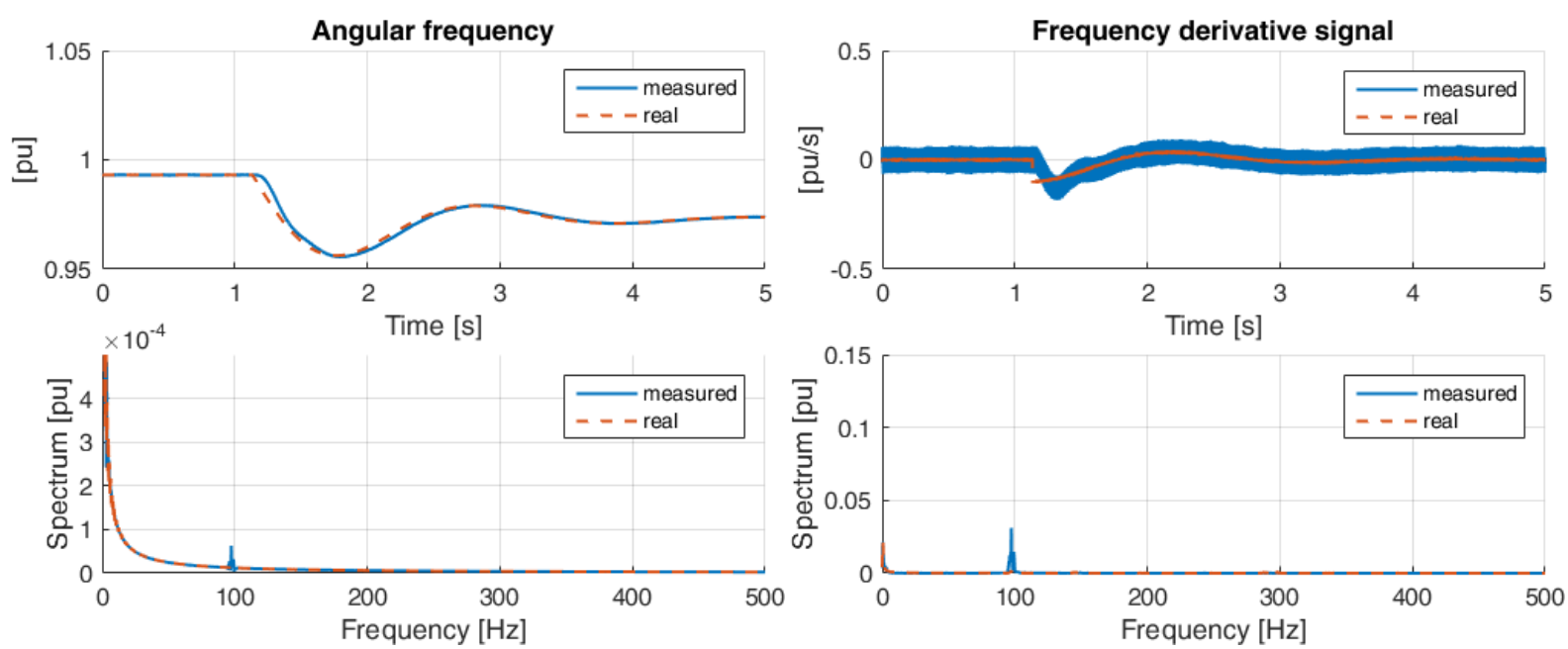

Figure 8: Angular frequency (a) and its derivative (b) obtained by a SO-SOGI scheme with fast FLL loop $\left(K_{F L L}=80 \mathrm{rad} / \mathrm{s}\right)$. High order terms are reduced with respect to Fig. 7, but the $100 \mathrm{~Hz}$ disturbance (produced by the negative sequence) remains significant in the derivative signal.
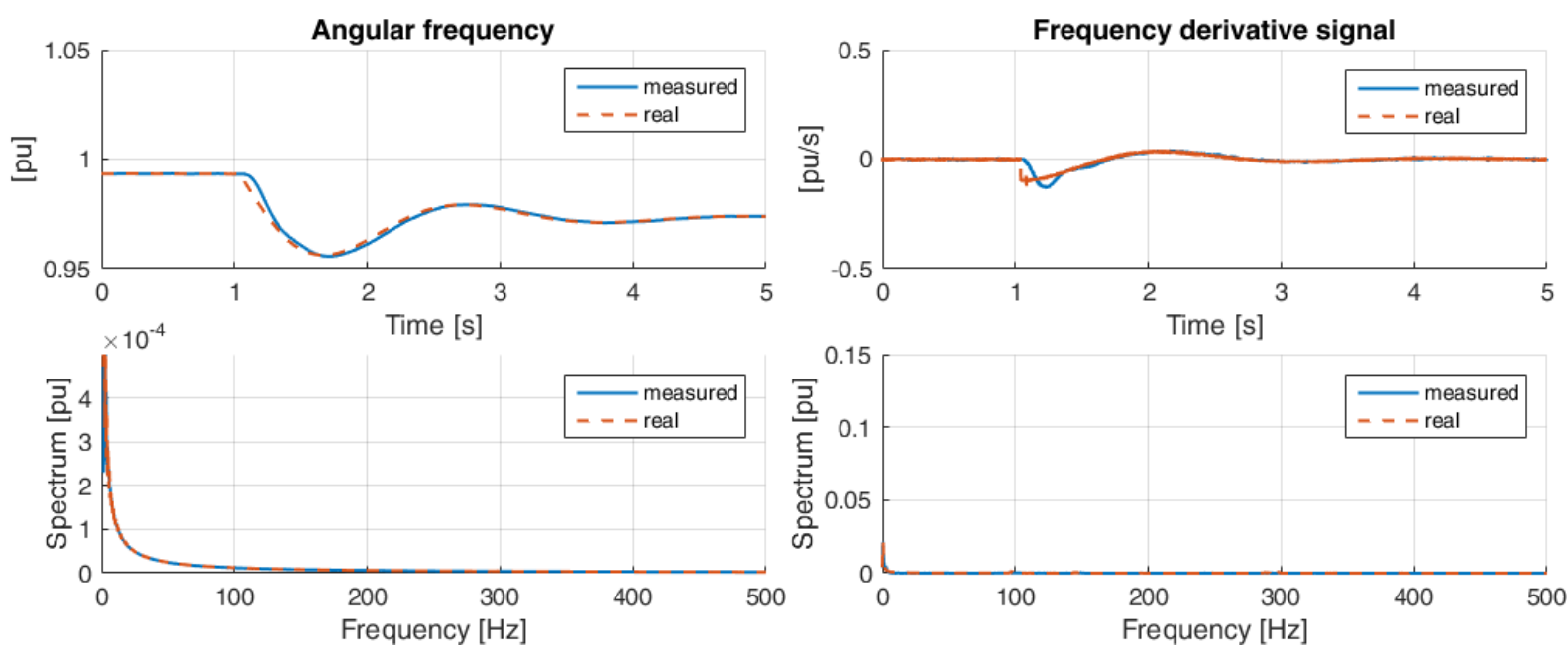

Figure 9: Angular frequency signal (a) and its derivative (b) with a SO-SOGI-N scheme (fast FLL). The reconstructed quantities match with the real ones: disturbance rejection and convergence time are acceptable. 
The convergence time associated to each estimator matches with the theoretical one $\left(t^{*} \simeq 5 / k_{F L L}\right)$ associated to the linearized FLL transfer function (19). Additionally, some Performance Indexes (PIs) have been identified to carry out a rigorous comparison between the algorithms. The considered PIs are reported in the following (20)-(23): according to their definitions the higher the value of each PI, the better the algorithm behaviour. Following aspects are taken into account in the PIs definition:

- Total harmonic distortion of the angular frequency at steady-state, including disturbances up to $500 \mathrm{~Hz}$ (20), where $\omega_{0}$ represents the steady-state value of the angular frequency.

- Harmonic content of the angular frequency derivative at steady-state, including disturbances up to $500 \mathrm{~Hz}(21)$.

- Integral absolute error between actual (real) and estimated (measured) angular frequency during the transient (22).

- Integral absolute error between actual (real) and estimated (measured) derivative during the imposed transient (23).

$$
\begin{array}{ll}
P I_{1}^{-1}=\sqrt{\sum_{h \neq 0}\left(\omega_{h}^{2} / \omega_{0}^{2}\right)-1} & P I_{2}^{-1}=\sqrt{\sum_{h \neq 0} \dot{\omega}_{h}^{2}} \\
P I_{3}^{-1}=\sum_{t=0}^{T=5 s}\left|\omega_{\text {real }}(t)-\omega_{\text {meas }}(t)\right| & P I_{4}^{-1}=\sum_{t=0}^{T=5 s}\left|\dot{\omega}_{\text {real }}(t)-\dot{\omega}_{\text {meas }}(t)\right|
\end{array}
$$

The numerical values of the Performance Indexes are reported in Table II. In order to produce equal combination of the single indexes, they have been normalized (in brackets) with respect to the maximum column value. A global performance index $P I_{G}^{\text {norm }}$ is obtained as the mean of the single normalized indexes for each estimator: this global index thus ranges in [0;1], with the higher end of the interval corresponding to the best performances. The analysis of the data in Table II reveals the outperformance of the proposed SO-SOGI-N scheme with respect to the other considered algorithms.

Table II: Performance Index (PI) comparison

\begin{tabular}{c|cccc|c}
\hline Estimator (Fig.) & $\boldsymbol{P I}_{\mathbf{1}}$ & $\boldsymbol{P I}_{\mathbf{2}}$ & $\boldsymbol{P I}_{\mathbf{3}}$ & $\boldsymbol{P I}_{\mathbf{4}}$ & $\boldsymbol{P I}_{\mathbf{G}}^{\text {norm. }}$ \\
\hline Slow DSOGI & $1.0 \cdot 10^{4}$ & $5.2 \cdot 10^{1}$ & $3.3 \cdot 10^{-2}$ & $5.5 \cdot 10^{-3}$ & 0.44 \\
(Fig. 6) & $(1.0)$ & $(0.22)$ & $(0.15)$ & $(0.39)$ & \\
Fast DSOGI & $3.0 \cdot 10^{3}$ & $5.3 \cdot 10^{0}$ & $2.2 \cdot 10^{-1}$ & $9.1 \cdot 10^{-4}$ & 0.35 \\
(Fig. 7) & $(0.30)$ & $(0.023)$ & $(1.0)$ & $(0.065)$ & \\
Fast SO-SOGI & $9.1 \cdot 10^{3}$ & $1.7 \cdot 10^{1}$ & $1.3 \cdot 10^{-1}$ & $2.6 \cdot 10^{-3}$ & 0.44 \\
(Fig. 8) & $(0.91)$ & $(0.074)$ & $(0.59)$ & $(0.18)$ & \\
Fast SO-SOGI-N & $1.0 \cdot 10^{4}$ & $2.3 \cdot 10^{2}$ & $1.3 \cdot 10^{-1}$ & $1.4 \cdot 10^{-2}$ & 0.90 \\
(Fig. 9) & $(1.0)$ & $(1.0)$ & $(0.59)$ & $(1.0)$ & \\
\hline
\end{tabular}

\section{Conclusions}

This paper proposes an extended estimator of the grid angular frequency and its derivative, in the perspective of fast regulation services provision to power networks. A detailed focus on the interaction of the developed architecture with the Frequency Locked Loop has been provided, also comparing the scheme with similar solutions available in literature. The experimental tests highlight the optimal performances of the proposed SO-SOGI-N scheme, both in terms of convergence speed and insensitivity to the network disturbances.

\section{Appendix: Derivation of the formulas used for sequences extraction}

Assume that it is possible to identify an hypothetic real-coefficients transfer functions $Z(s)$ characterized by a constant magnitude profile $\left|Z\left(j \omega_{s}\right)\right| \equiv 1$ and by a constant phase displacement equal to $90^{\circ}$ lagging in the whole frequency range $\measuredangle Z\left(j \omega_{s}\right)=-90^{\circ}$. From (1), the $\alpha \beta$ components of the positive and negative voltage sequences are obtained, taking into account that:

$$
v_{\alpha}=v_{\alpha}^{+}+v_{\alpha}^{-} \quad v_{\beta}=v_{\beta}^{+}+v_{\beta}^{-}
$$




$$
\begin{aligned}
& v_{\alpha}^{+}=\operatorname{Re}\left\{\sum_{h=1}^{N+} \hat{V}_{h}^{+} e^{j h \omega_{1} t}\right\}=\sum_{h=1}^{N+}\left|\hat{V}_{h}^{+}\right| \cos \left(h \omega_{1} t+\varphi_{h}^{+}\right) \\
& v_{\beta}^{+}=\operatorname{Im}\left\{\sum_{h=1}^{N+} \hat{V}_{h}^{+} e^{j h \omega_{1} t}\right\}=\sum_{h=1}^{N+}\left|\widehat{V}_{h}^{+}\right| \sin \left(h \omega_{1} t+\varphi_{h}^{+}\right) \\
& v_{\alpha}^{-}=\operatorname{Re}\left\{\sum_{h=1}^{N-} \hat{V}_{h}^{-} e^{-j h \omega_{1} t}\right\}=\sum_{h=1}^{N-}\left|\widehat{V}_{h}^{-}\right| \cos \left(-h \omega_{1} t+\varphi_{h}^{-}\right) \\
& v_{\beta}^{-}=\operatorname{Im}\left\{\sum_{h=1}^{N-} \hat{V}_{h}^{-} e^{-j h \omega_{1} t}\right\}=\sum_{h=1}^{N-}\left|\hat{V}_{h}^{-}\right| \sin \left(-h \omega_{1} t+\varphi_{h}^{-}\right)
\end{aligned}
$$

As a consequence of the characteristics of the transfer function $Z(s)$, it is possible to obtain:

$$
\begin{aligned}
& z v_{\alpha}=\mathcal{L}^{-1}\left\{Z(s) V_{a}(s)\right\}=z v_{\alpha}^{+}+z v_{\alpha}^{-} \quad z v_{\beta}=\mathcal{L}^{-1}\left\{Z(s) V_{\beta}(s)\right\}=z v_{\beta}^{+}+z v_{\beta}^{-} \\
& z v_{\alpha}^{+}=\sum_{h=1}^{N+}\left|\widehat{V}_{h}^{+}\right| \cdot \cos \left(h \omega_{1} t+\varphi_{h}^{+}-\frac{\pi}{2}\right) \quad z v_{\beta}^{+}=\sum_{h=1}^{N+}\left|\widehat{V}_{h}^{+}\right| \cdot \sin \left(h \omega_{1} t+\varphi_{h}^{+}-\frac{\pi}{2}\right) \\
& z v_{\alpha}^{-}=\sum_{h=1}^{N-}\left|\widehat{V}_{h}^{-}\right| \cdot \cos \left(-h \omega_{1} t+\varphi_{h}^{-}+\frac{\pi}{2}\right) \quad z v_{\beta}^{-}=\sum_{h=1}^{N-}\left|\hat{V}_{h}^{-}\right| \cdot \sin \left(-h \omega_{1} t+\varphi_{h}^{-}+\frac{\pi}{2}\right)
\end{aligned}
$$

Analytically manipulating (30) and (31) and combining them with the definition in (25)-(28), leads to the following equivalences: $z v_{\alpha}^{+}=v_{\beta}^{+}, z v_{\alpha}^{-}=-v_{\beta}^{-}, z v_{\beta}^{+}=-v_{\alpha}^{-}, z v_{\beta}^{-}=v_{\alpha}^{+}$. Thus:

$$
z v_{\alpha}=z v_{\alpha}^{+}+z v_{\alpha}^{-}=v_{\beta}^{+}-v_{\beta}^{-} \quad z v_{\beta}=z v_{\beta}^{+}+z v_{\beta}^{-}=-v_{\alpha}^{+}+v_{\alpha}^{-}
$$

Equations (24) and (32) define a $4^{\text {th }}$ order determined linear system in the variables $\left(v_{\alpha}, z v_{\alpha}^{\prime}, v_{\beta}, z v_{\beta}^{\prime}\right)$, that can easily lead to the expressions of positive and negative sequences reported in (7). From a rigorous point of view, the characteristics of the ideal transfer function $Z(s)$ cannot be obtained from a real-coefficient rational expression; nevertheless, the combination of $D(s)$ and $Q(s)$ meet the identical magnitude requirement and the $\pi / 2$ shifting at the resonance point, thus correctly approximates $Z(s)$ behaviour, enabling the sequences extraction by means of (7). Far from the resonance condition, the sequence extraction may be affected by the non-ideal behaviour of $D(s)$ and $Q(s)$.

\section{References}

[1] J. Rocabert, A. Luna, F. Blaabjerg and P. Rodríguez, "Control of Power Converters in AC Microgrids", in IEEE Transactions on Power Electronics, vol. 27, no. 11, pp. 4734-4749, Nov. 2012.

[2] A. Bolzoni, G. M. Foglia, L. Frosio, M. F. Iacchetti, R. Perini, "Impact of Line and Control Param. on Droop Stability in Inverters for Distributed Gen." in IEEE Trans. on Smart Grid, vol. 9, no. 6, Nov. 2018.

[3] A. Bolzoni, C. Terlizzi and R. Perini, "Analytical Design and Model. of Power Conv. Equipped with Synthetic Inertia Control", 20th Europ. Conf. on Power Electr. and Applic. (EPE'18 ECCE), Riga, Latvia, 2018.

[4] J. Fang, H. Li, Y. Tang and F. Blaabjerg, "Distributed Power System Virtual Inertia Implemented by GridConnected Power Conv.", in IEEE Trans. on Power Electronics, vol. 33, no. 10, pp. 8488-8499, Oct. 2018.

[5] M. F. M. Arani and E. F. El-Saadany, "Implementing Virtual Inertia in DFIG-Based Wind Power Generation", in IEEE Transactions on Power Systems, vol. 28, no. 2, pp. 1373-1384, May 2013.

[6] P. Castello, J. Liu, C. Muscas, P. A. Pegoraro, F. Ponci, A. Monti, "A Fast and Accurate PMU Algorithm for P+M Class Meas. of Synchrophasor and Frequ.", IEEE Trans.on Instrum.and Meas., vol.63, no.12, Dec. 2014

[7] Z. Xin, X. Wang, Z. Qin, M. Lu, P. C. Loh and F. Blaabjerg, "An Improved Second-Order Generalized Integrator Based Quadrature Signal Generator", in IEEE Trans. on Power Electr., vol. 31, no. 12, Dec. 2016.

[8] R. Teodorescu, M. Liserre, P. Rodríguez, "Grid converters for photovoltaic and wind power systems", John Wiley and Sons, 2010, chapter 8. 